\title{
Myocardial Transmural Electrical Disruption Affects Electrogram Pattern
}

\author{
Mirabeau Saha ${ }^{1,2}$, Caroline Roney ${ }^{3}$, Hubert Cochet $^{2}$, Steven Niederer ${ }^{3}$, Edward Vigmond ${ }^{2}$, \\ Stanley Nattel ${ }^{1,2}$ \\ ${ }^{1}$ Department of Medicine and Research Center, Montreal Heart Institute, Montreal, QC, Canada \\ ${ }^{2}$ IHU Liryc, Electrophysiology and Heart Modeling Institute, Fondation Bordeaux Université, \\ Pessac, France \\ ${ }^{3}$ Department of Biomedical Engineering, King's College London, London, United Kingdom
}

\begin{abstract}
Myocardial structural remodeling leads to atrial fibrillation $(A F)$. Interstitial collagen deposits remodel myocardium, causing disruption of electrical propagation between the endocardial and the epicardial layers, and affecting propagation within layers as well. How these changes manifest on the electrogram (EGM) is unclear. Here we investigate the consequences of epicardiumendocardium electrical dissociation on EGMs. Left atrial patient-specific bilayer computational models were constructed using MRI from AF patients $(n=11)$. Interstitial collagen was incorporated as microstructural discontinuities within layers and causing transmural dissociation in the high-fibrosis areas. The models where the fibrosis was not included were considered as controls. Changes in the unipolar EGM characteristics were computed. A propagation delay was observed between both layers. No monotonic linear relationship between control and fibrotic EGMs was found. With the collagen deposits, amplitude decreases and increased fractionation on EGMs were significant. EGM area also tended to become smaller, and duration and waveform asymmetry were affected. In conclusion, measurements of EGM morphology can be used together with clinical imaging data to distinguish between different substrate modifications, and better select ablation targets.
\end{abstract}

\section{Introduction}

Atrial fibrillation (AF) is the most common arrhythmia seen in clinical practice. It affects over 2.5 million people in USA [1]. Its incidence is increasing year by year, and an epidemic of $\mathrm{AF}$ is forecast within the next 10 to 20 years because of the worldwide aging of the population [2]. AF initiation and $\mathrm{AF}$ perpetuation mechanisms have been shown to be related to remodelling of atrial tissue properties, including atrial fibrosis. Myocardial remodeling includes collagen deposition, fibroblast coupling changes, myocyte replacement, and electrical remodeling. Fibrosis causes myocardial structural alteration and microstructural discontinuities with uncoupling of cells, leading to epicardium-endocardium electrical disruption [3]. This is turn affects the propagation of the action potential signal across atrial tissue $[4,5]$. The measurement of electrograms (EGMs) on the atrial surface, reflecting action potential propagation, is helpful to identify the target for catheter ablation therapy of AF. The presence of microstructural discontinuities leads to wavefront collisions which can produce complex fractionated atrial EGMs during sinus rhythm. These complex fractionated atrial EGM areas are often targeted during catheter ablation therapy $[6,7]$. Although fibrosis has been associated with the presence of fractionated EGMs, determining the fibrosis nature by simple analysis of EGM characteristics is challenging [5]. Here, we sought to determine the differences in $\mathrm{AF}$ EGMs in normal versus fibrotic atria (interstitial collagen deposits) with electrical disruption between the epicardial and endocardial layers.

\section{Methods}

\subsection{Models}

We used eleven patient-specific left atrial bilayer computational models from our previous work [8]. As described in that work, the bilayer models were constructed using late gadolinium enhancement (LGE) magnetic resonance imaging (MRI) data from patients with persistent AF (Fig. 1(A)). The LGE-MRI data were segmented and meshed to create finite element meshes with fiber direction and LGE distributions (Fig. 1(B)) suitable for electrophysiology simulations. Each left atrial bilayer model consisted of linearly coupled endocardial and epicardial layers. LGE distributions were used as a scaling factor for the probability of interstitial collagen deposition intensity (fibrosis). The fibrosis content varied from $14.5 \%$ to $42.2 \%$ for the eleven patients. To model the in-vivo collagen deposition (which is known to be greater in the endocardial layer), 
(A)

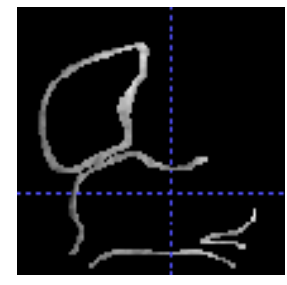

(B)

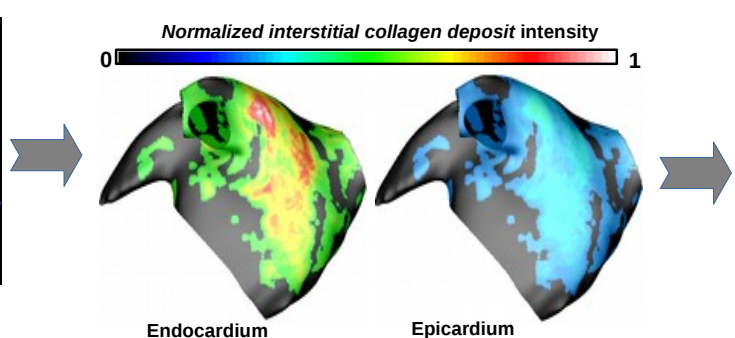

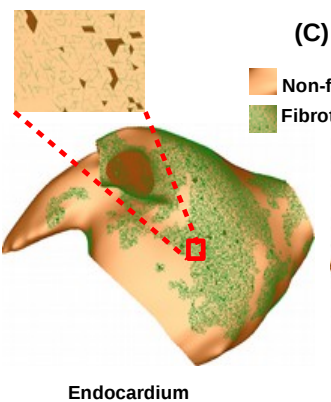

(C)

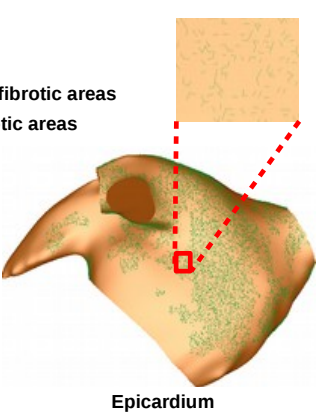

Figure 1: Model construction: (A) Clinical imaging data of a patient with persistent AF. (B) Patient specific left atrium meshes with interstitial collagen deposit distribution. (C) Microstructural discontinuity distribution implemented on the epicardial and endocardial layers, representing interstitial collagen deposit. The difference in fibrosis distribution between both layers is reflected on these figures.

LGE intensity in the epicardia layer was set at $40 \%$ of the endocardial layer value. As shown in figure 1(C), the effects of collagen were modeled as microstructural discontinuities using the edge-splitting method [9]. Moreover, the linear coupling between layers were disrupted in the fibrotic area. For each patient, the AF non-fibrotic model was taken as the control. In total, there were 22 left atrial bilayer AF models, 11 with fibrosis and the rest were controls.

\subsection{Simulations}

Each model was simulated for one beat during sinus rhythm using the monodomain formulation, solved with the Cardiac Arrhythmia Research Package (CARP) simulator [7]. The stimulus was initiated on the point of attachment of Bachmann's bundle. For a given instant, the EGM at electrode's position $\mathbf{p}$ was computed using the following equation (1).

$$
\phi(\mathbf{p})=\int_{\boldsymbol{\Omega}} \frac{\nabla \mathbf{V}_{\mathbf{m}}\left(\mathbf{p}^{\prime}\right)\left(\mathbf{p}-\mathbf{p}^{\prime}\right)}{4 \pi \sigma_{\mathbf{e}}\left\|\mathbf{p}-\mathbf{p}^{\prime}\right\|^{3}} \mathbf{d} \boldsymbol{\Omega}
$$

where $\sigma_{\mathbf{e}}$ is extracellular conductivity $\mathbf{d} \Omega, \mathbf{V}_{\mathbf{m}}$ is transmembrane voltage, $\mathbf{p}$ is the position of the electrode and $\mathbf{p}^{\prime}$ is the position of $\mathbf{d} \boldsymbol{\Omega}$. Unipolar EGMs were recorded in the fibrotic models at the same positions as for the control models.

\subsection{Signal analysis}

A clinical temporal filtering of polynomial order 3 was applied to all EGMs. Various EGM characteristics were used to evaluate the change from control to fibrotic cases. Let $\mathrm{R}$ and $\mathrm{S}$ be the absolute maximum amplitudes of the positive deflection and the negative deflection of an EGM, respectively. The peak to peak amplitude and asymmetry of an EGM are giving by equations (2) and (3) [5], respectively.

$$
\begin{gathered}
\text { Amplitude }=\mathbf{R}+\mathbf{S} \\
\text { Asymmetry }=(\mathbf{R}-\mathbf{S}) /(\mathbf{R}+\mathbf{S})
\end{gathered}
$$

The amplitude indicates the magnitude of the EGM and the asymmetry reflects its mean sign (positive or negative). The number of deflections are defined as the number of local extrema whose local amplitude is larger than $1 \%$ of the amplitude. For each patient, the fractionation duration was computed by setting a threshold of $2.5 \%$ of the amplitude to define the onset-time and the end-time of the wavefront on each electrode. The correlation coefficient between the control EGM and the fibrotic one was computed using Pearson formulation. EGM area was defined as the time trapezoidal integral between the onset-time and the end-time of the signal.

\section{Results and Discussion}

Analysis of the electrical wave dynamics showed that the modelled collagen deposits caused perturbations and local irregularities at the microscale on the wavefront, and slowed down propagation of the action potential as observed in figure 2(A). Irregularities were very pronounced in the high fibrotic-density regions. Since the endocardium was more fibrotic than the epicardium, the wavefront propagation was slower and more discrete on the endocardium. The mean propagation delay between layers was $17 \pm 7$ $\mathrm{ms}$ for the eleven patients. Wavefront collisions were more common in the endocardium compared to the epicardium. Activation delay (and similar behaviors) between epicardium and endocardium in structurally remodeled atrial tissue was found in a clinical study by Eckstein et al [10]. This supports the veracity of our mathematical study. A sample of unipolar EGM plots from patient 11 is represented in figure 2(B) for a single peaced beat. Differences between EGM morphologies from control and fibrotic models are observed. The characterization of the 
(A)

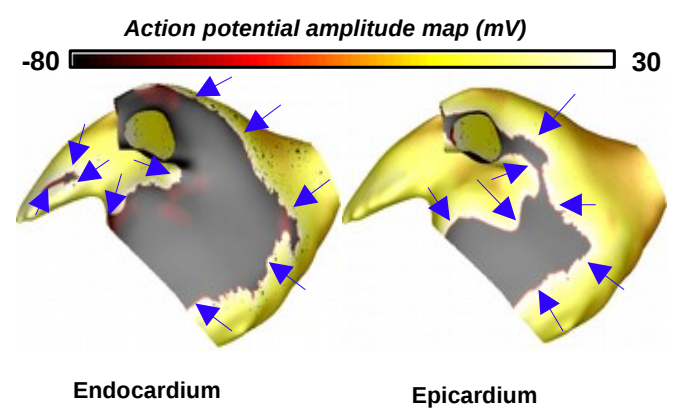

(B)

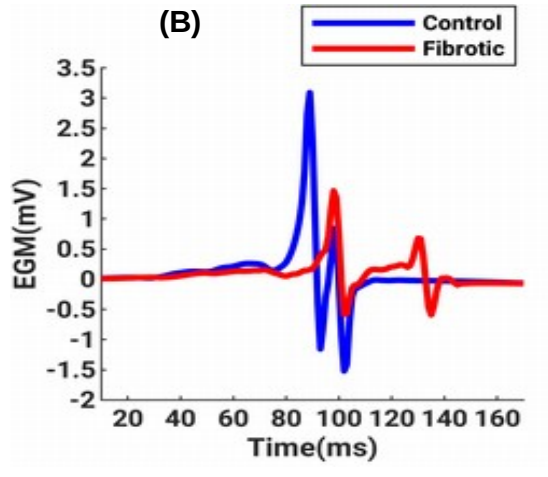

Figure 2: Electrical wave propagation and EGM: (A) Action potential propagation in endocardial and epicardial layers $100 \mathrm{~ms}$ after stimulation. Arrows indicate the wave front propagation direction. A delay is observed in the endocardial layer. (B) A sample of unipolar EGM from the $11^{\text {th }}$ patient. Differences between EGM morphologies from control and fibrotic models are observed.

differences is shown in figure 3 for all the patients. For each patient, quantities are represented relative to the corresponding control. According to the correlation coefficient analysis (see figure 3(F)), there was no perfect downhill or uphill linear relationship between control model EGMs and the corresponding ones in the fibrotic models. In most cases, there was a weak or moderate uphill linear relationship between both EGMs. But some patients, such as patient 2 , presented almost no relationship between control and fibrotic EGMs (the coefficient of correlation is almost zero). We observe in figure 3(A) a decrease in EGM amplitude from the control to the fibrotic model due to collagen deposit. For the eleven patients, the maximum decrease was $61.33 \%$ and the minimum was $11.68 \%$. The mean amplitude decrease was $39 \pm 14 \%(p<0.05)$ over all patients. As well as slowing down propagation, fibrosis tends to modify EGM duration. As shown in figure 3(B), while 6 out of 11 patients experienced a decrease in EGM duration, the remaining 5 patients experienced significant increases. Overall, the duration increase was between $-34.08 \%$ and $+112.4 \%$ with a mean of $24 \pm 44 \%$ $(p=0.18)$ for the 11 patients. Due to the discrete nature of impulse propagation, significant fractionation appears on the fibrotic EGM despite the temporal filtering applied. As shown in figure 3(C), we observed a general increase of the number of deflections in the fibrotic models, ranging from $40 \%$ to $200 \%$ with a mean of $114 \pm 54 \%$ $(p<0.05)$ over all patients. EGM amplitude reduction due to collagen deposits led to the diminution of EGM area (as illustrated in figure 3(D)) from $40 \pm 9 \mu V s$ for the control models to $33 \pm 10 \mu V s$ for the fibrotic models $(p=0.08)$, with patient 4 being an exception in whom an increase was observed. Figure 3(E) shows that collagen deposit mainly caused an absolute decrease in waveform asymmetry (7 cases out of 11). However, in few cases, we observed an absolute increase of the asymmetry ( 3 cases out of 11) due to the presence of collagen. Sometimes (1 case out of 11), waveform asymmetry was reversed from the control to the fibrotic model. It has been suggested that asymmetry is related to the conduction differences between layers [11] and according to Jacquemet et al [12], anisotropy, wavefront collisions and curvature (affected by collagen deposit) also influence EGM asymmetry. Our results are consistent with these ideas.

\section{Conclusions}

In this work using left atrial bilayer computational models from persistent AF patients, we have analyzed the differences between the morphologies of the EGMs recorded on the non-fibrotic atria and those recorded on the atria with interstitial collagen deposits causing electrical disruption between atria epicardial and endocardial layers. The results showed striking differences between the two signals for relevant characteristics. However, we need to extend our work to better understand these differences. By allowing for improved detection of regions of dense interstitial collagen deposit in the atria, through the appropriate analysis of clinical EGMs recorded during sinus rhythm in AF patients, such studies may help to enhance the precision and success of clinical substrate-guided ablation for AF.

\section{Acknowledgments}

The work was supported by the "Fondation de la recherche médicale" (FRM) grant SPF20160936220. It received financial support from the National Research Agency (ANR), grant ANR-10-IAHU-04. Computer time was provided by the computing facilities Marconi at CINECA, Italy. 

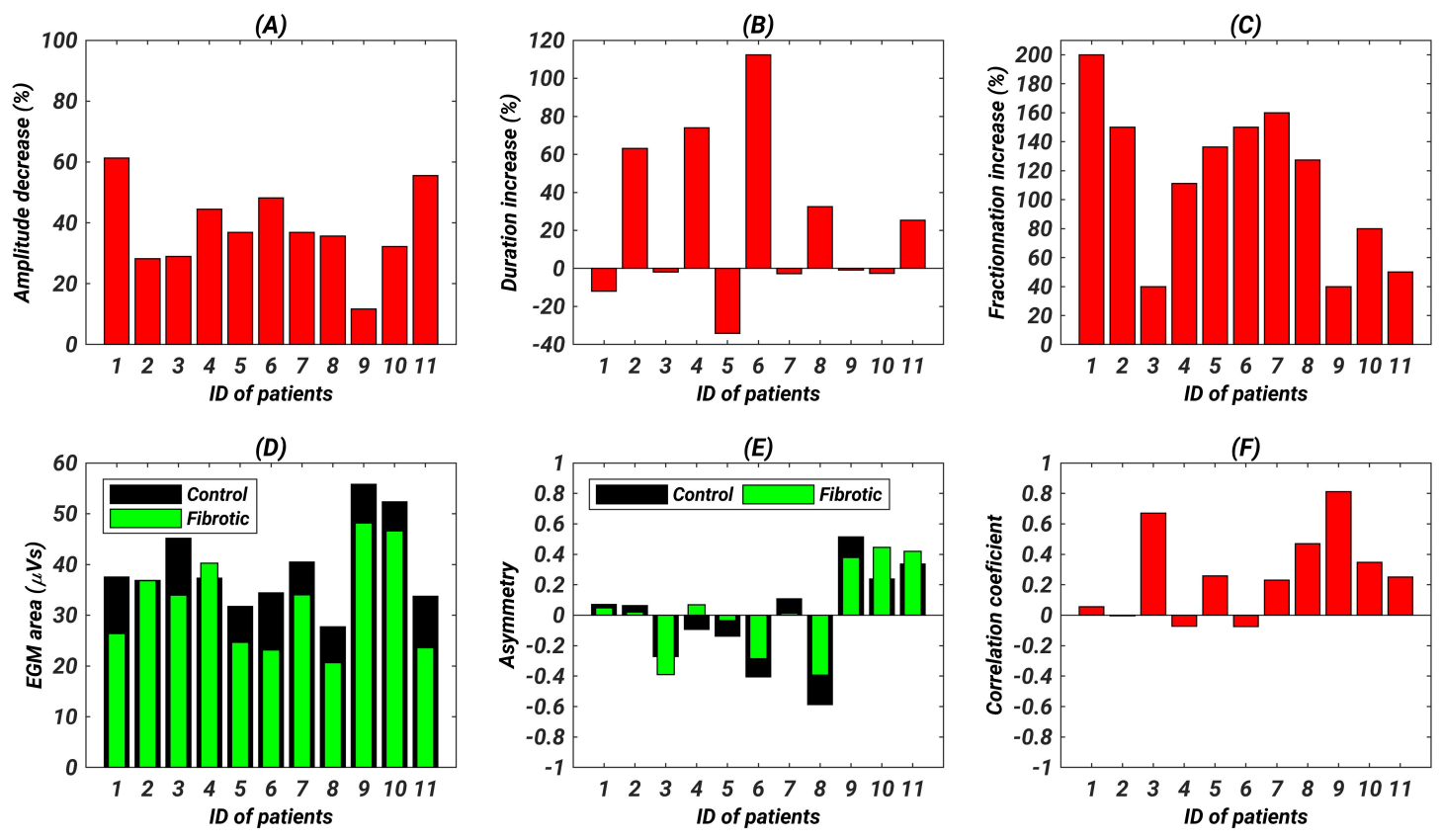

Figure 3: EGMs morphology analysis: (A) Amplitude decrease, (B) Changes of EGM duration, (C) Increase of EGM fractionation, (D) EGMs area, (E) Asymmetry and (F) Correlation coefficient between control and fibrotic EGM signals.

\section{References}

[1] McManus DD, Rienstra M, Benjamin EJ. An update on the prognosis of patients with atrial fibrillation. Circulation 2012;126(10):e143-e146.

[2] Morillo CA, Banerjee A, Perel P, Wood D, Jouven X. Atrial fibrillation: the current epidemic. Journal of geriatric cardiology JGC 2017;14(3):195.

[3] Horn MA, Trafford AW. Aging and the cardiac collagen matrix: Novel mediators of fibrotic remodelling. Journal of molecular and cellular cardiology 2016;93:175-185.

[4] Oh S, Rhee T, Lee S, Cha M, Choi E. P3630association of complex fractionated electrograms with atrial myocardial thickness and fibrosis: Insights from pathologic analyses of canine hearts. European Heart Journal 2017;38(suppl_1).

[5] Jacquemet V, Henriquez CS. Genesis of complex fractionated atrial electrograms in zones of slow conduction: a computer model of microfibrosis. Heart rhythm 2009;6(6):803810.

[6] Saghy L, Callans DJ, Garcia F, Lin D, Marchlinski FE, Riley M, Dixit S, Tzou WS, Haqqani HM, Pap R, et al. Is there a relationship between complex fractionated atrial electrograms recorded during atrial fibrillation and sinus rhythm fractionation? Heart rhythm 2012;9(2):181-188.

[7] Vigmond EJ, Hughes M, Plank G, Leon LJ. Computational tools for modeling electrical activity in cardiac tissue. Journal of electrocardiology 2003;36:69-74.

[8] Roney CH, Williams SE, Cochet H, Mukherjee RK, ONeill L, Sim I, Whitaker J, Razeghi O, Klein GJ, Vigmond EJ, et al. Patient-specific simulations predict efficacy of ab- lation of interatrial connections for treatment of persistent atrial fibrillation. EP Europace 2018;20:iii55-iii68.

[9] Roney CH, Bayer JD, Zahid S, Meo M, Boyle PM, Trayanova NA, Haïssaguerre M, Dubois R, Cochet H, Vigmond EJ. Modelling methodology of atrial fibrosis affects rotor dynamics and electrograms. EP Europace 2016; 18(suppl_4):iv146-iv155.

[10] Eckstein J, Maesen B, Linz D, Zeemering S, Van Hunnik A, Verheule S, Allessie M, Schotten U. Time course and mechanisms of endo-epicardial electrical dissociation during atrial fibrillation in the goat. Cardiovascular research 2010;89(4):816-824.

[11] Houben RP, de Groot NM, Smeets JL, Becker AE, Lindemans FW, Allessie MA. S-wave predominance of epicardial electrograms during atrial fibrillation in humans: indirect evidence for a role of the thin subepicardial layer. Heart Rhythm 2004;1(6):639-647.

[12] Jacquemet V, Virag N, Ihara Z, Dang L, Blanc O, Zozor S, VESIN JM, Kappenberger L, Henriquez C. Study of unipolar electrogram morphology in a computer model of atrial fibrillation. Journal of cardiovascular electrophysiology 2003;14:S172-S179.

Address for correspondence:

Mirabeau Saha

Montreal Heart Institute, 5000 Rue Bélanger, S4500, Montreal, Quebec, H1T 1C8, Canada mirabeau.saha@mail.mcgill.ca 